\title{
Investigating the Use and Non-Use of Online Information Resources Among Cancer Patients: A Qualitative Study
}

\author{
Zahraa A. Abul ${ }^{1}$ \\ Abbas Al-Salman ${ }^{1}$ \\ ${ }^{1}$ Library information systems department, graduate studies at Kuwait university, Kuwait
}

\begin{abstract}
This research focuses on examining the use and non-use of information resources among cancer patients. Non-seekers' information-seeking behavior is analyzed in this research to determine the causes and symptoms of their non-seeking of health knowledge. Cancer patients use a variety of information resources such as the Internet, social media, and medical databases, in order to satisfy their needs. Studies showed that patients' knowledge of their cancer stage might help them in making their care and treatment choices. Focus groups are commonly used in health research to explore the perspectives of patients or health care professionals; relatively few studies consider methodological aspects in this specific context. The purpose of this research is to investigate the information needs of cancer patients, and to find out reasons for the lack of cancer stage knowledge and desire for information after diagnosis. Expected findings include personal, situational, and psychological reasons, such as: low health literacy, low usage of health information, health instructions and the ways to increase patient awareness of the benefits and ways of seeking information.
\end{abstract}

Key words: Kuwait: Library information science: information resources: Health information 


\section{INTRODUCTION}

Many studies in the social informatics field assumes that using technology while seeking information raises the user's information literacy and fulfills their information needs. A rapid increase in the use of information and communication technologies (ICTs) in recent decades is an enormous contributing factor in the development of some novel clinical and public health intervention strategies at every level, including primary prevention, screening, early diagnosis, treatment, survivorship, and end-of-life care (Coughlin et al., 2016). ICTs are broadly defined as technologies used to communicate, manipulate and store data by electronic means. These include email, short message service (SMS) text messaging, video chat, web-based social media, as well as all the different computing devices that perform a wide range of communication and information functions. Furthermore, different factors affect the information-seeking process for cancer patients; these include factors as wide-ranging as education, age, race, health status, employment status, and type of cancer. ICTs might be beneficial for cancer patients, when defined as the sources from which they seek information related to their disease. There has been an increase in the number of publications about public health uses of social networking sites in the past few years (Capurro et al, 2014). This growing body of research regarding the impact and utility of ICTs emphasizes the importance of and potential uses that such technologies add to the patient-physician relationship.

On the other hand, perspectives toward the preferred resource consulted by cancer patients vary, as demonstrated in a study by Rainie (2000); this concluded that online sources on the internet are the preferred resource, but that there is a group of cancer patients who refuse or avoid seeking and receiving health information. Other sources of information for cancer patients are: health care providers, specialists (such as psychologists), libraries, health databases, and face-toface communication with doctors. As we saw from our visit to "Sidra Center", which is classified as a health care center, it is functioning as a professional medical resource for cancer patients. Centers such as this play a crucial role in providing cancer patients with the information they seek related to their cancer stage, and that which would help them in making health decisions. As a result, patient education and health knowledge have shown marked impacts on cancer patients' health outcomes over the years - on both the physical and psychological sides.

This research aims to study the reason behind the lack of desire to seek, or avoidance of, information among some cancer patients. We take into account a wide range of factors, including socioeconomics and demographics. Our research question focuses on the type of resources sought, channels used for seeking information, and the reasons behind the lack of desire to search for information. Cancer information-seeking behavior is being changed with both advanced cancer treatment and improved health literacy. Many cancer patients want information, but not all have the desire to seek or receive this type of information - especially after diagnosis. Seekers are defined as cancer patients who actively seek information online; they are often educated and younger. On the other hand, non-seekers are the other type of cancer patients that lack the desire to search for information online - although they may use other information resources. They might prefer to receive information from their doctors, physicians, health care providers, and support group members. The non-seeking behavior occurs due to a number of reasons, as can be seen in the literature of cancer patient information-seeking behavior. Lydon et al. (2000) pointed out that non-seeking cancer patient's attitudes, in the process of coping with 
their illness, were expressed through lacking the desire for knowing or obtaining related information. The underlying reasons for this issue vary; they can be categorized into three groups: faith, hope, and charity. Furthermore, some patients intentionally avoid information that may cause them anxiety or stress, a behavior characterized as "blunting", in opposition to "monitoring" or active information seeking" (Ramanadhan, 2006).

It was found that cancer patients avoid health information for a number of reasons: because they have enough trust in their doctor's instructions; because avoidance might reduce anxiety; because knowing too much information may mislead them and lead to overload; because they fear the unreliability of some health information online; because they are protecting themselves from danger of violating their privacy; and even, in some cases, rejecting the received information because it is difficult to carry out. Accordingly, our objective with this research is to understand the non-seekers, who for different reasons do not search for information at times at which it would be expected. For this reason, a group of five cancer survivors will be studied to reach the aim of this research. Questions that underpin our research are:

What are the searching skills of cancer patients and non-seekers?

What are the type of information resources that present cancer patient's preferences?

What are the reasons cancer patients refuse to seek information? Do cancer patients lack the desire for information before diagnosis as well, or only after?

\section{LITERATURE REVIEW}

\section{Uses of ICT for Cancer Patients}

The revolutionary progression in information and communication technologies (ICTs) and their rapid deployment are prompting a significant effect on individuals, foundations, and communities. The spread of the Internet has prevailed, interweaving people and organizations, facilitating rapid and wide dissemination of information across geographic, temporal, and disciplinary boundaries (Viswanath et al. 2012). Searching for health information online now ranks third among online activities, with $59 \%$ of all adults searching for health information at some point (Fox, 2012). Similarly, Ramanadhan \& Viswanath (2006) mentioned that metaanalysis of studies of cancer patients using the Internet concluded that about $39 \%$ of those with cancer are using the Internet. Communication technologies that allow users to access social media sites (e.g., computers, tablets, smartphones) are becoming progressively more accessible (Chou et al. 2013).

Due to social media representing one effective interface when seeking information, it is essential to examine how social media may bring cancer-related information and interventions to populations affected by the digital divide and subject to disparities (Viswanath et al. 2013). The marriage of consumer informatics with health informatics has induced an unusual level of good faith on how ICTs could be utilized to propel preventive health and enhance the quality of care, ultimately leading to improved health results (Viswanath et al. 2013). 


\section{Lack of desire to seek information}

Information-seeking is a critical concept in health communication, in light of the growing trend among patients, advocates, and researchers to move away from the paternalistic model of patient-provider communication, toward more educated or shared decision-making (Rimer et al. 2004). Despite recent reports about increased interest in health information in the general public, and the assumption that information-seeking is essential, not all individuals diagnosed with a severe condition actively seek information from sources other than their healthcare suppliers (Czaja et al. 2003; Rees \& Bath 2001). Similarly, Ramanadhan \& Vswanath (2006) demonstrated that not all individuals diagnosed with a severe condition actively seek information from sources other than their healthcare suppliers. Some patients intentionally avoid information that may cause them anxiety or stress - a behavior characterized as "blunting", and one which works in opposition to "monitoring" or active information-seeking (Rees \& Bath, 2001). For example, one-third of patients in a study reported avoiding or distracting themselves from health information when they felt that the outcome could not be altered (Miller, 1987). Despite reports of active information-seeking by individuals, either through their efforts or their social networks, some do not obtain information except for what they may get from their providers. In a recent study of women with breast cancer, focus group participants who did not seek out information noted that they did not want to worry, were frightened, or felt that information was negative and depressing (Rees \& Bath, 2001). Besides, Zilinski (2010) pointed out that numerous cancer patients do not efficiently seek information, but would rather give their physicians a chance to provide information about their disease instead.

In one study, it was concluded that patients perhaps had made efforts to obtain as much information as possible, "but the remaining 11 patients reported minimal efforts to obtain information additional to that offered by hospital staff. All the interviews revealed variability in attitude towards further information: patients did not want information about everything all of the time, but, at different times since their diagnosis, had wanted more or less information about particular aspects of their condition and its treatment" (Leydon et al., 2000). To differing degrees, patients showed faith in their specialists, and this added to their state of mind toward seeking information beyond that volunteered by health professionals in regular interactions.

Faith was linked with the view that medical knowledge was difficult to understand. This was especially the case among older patients, who - because they felt their knowledge and understanding of medicine was limited - believed that additional searching could be risky and exacerbate an already difficult situation. To return to the Leydon et al. (2000) study: "Belief in the maxim that 'doctor knows best' sometimes negated the perceived value of additional information, and patients believed that more information could confuse their situation. Having faith in their specialists' ability to successfully deploy what was often perceived as impressive and modern medical technologies often precluded information-seeking." The expression of hope often involved showing an overcoming face to others, and this itself could make it hard to discuss or seek information regarding cancer. Getting on with life and keeping up a positive and inspirational standpoint was seen as the approach to deal with disease that was most respected by hospital staff, friends, and family (Leydon et al., 2000). Also, a study reported that patients with low health literacy are faced with unacceptable or technical terms, complex thoughts and 
different choices, all of which can be overpowering. With coping being the primary aim, patients may diminish their decision burden using potentially maladaptive strategies such as information avoidance or keeping up an aloof approach. Further to this, patients with low literacy levels might not be aware of their informational deficits or the need to get more information to make decisions about their medical care, resulting in a lack of interest or desire to get more information (Costas et al. 2013). Similarly, Zilinski (2010) indicates that the level of education has been found to be a strong factor as to why some seekers might choose to avoid information and become non-seekers. Patients were also mindful that literature produced for patients "in general" was not significantly relevant to every individual, and the difficulty of perceiving information irrelevant to their situation was frustrating (Leydon et al., 2000).

\section{The motivation for seeking information}

Women tend to be more active seekers of health information in general - and around cancer specifically (Boudioni et al. 1999). Specific racial and ethnic groups, such as African-Americans, are thought to be less active information-seekers, but mixed results are found once other sociodemographic variables are controlled for (Matthews et al., 2002). Overall, younger patients tend to seek out more cancer information (and from a broader array of sources) than older patients. However, it is important to note that both groups report interest in being well-informed (Pinquart \& Duberstein, 2004). Information-seeking is impacting cancer patients' outcomes in some ways. The more informed patients participate in decision-making and report higher satisfaction with treatment approaches, which in turn is significantly associated with higher emotional, social, and cognitive functioning, and lower reports of side effects (Schou et al., 2005). Well-informed patients are also better able to cope with the diagnosis, treatment, and survivorship phases, and also with the effects of these phases on quality of life (Rutten et al., 2005). Patients and their families often cite physicians and friends as their preferred sources of information (Czaja et al., 2003; Rees \& Bath, 2000). However, patients increasingly are paying more attention to cancer information provided by professional societies, libraries, others in their social networks, and the Internet; and interestingly, gender mediates the choice of sources, age, and education (Carlsson, 2000; Rutten et al. 2005; Serin et al. 2004). A review of published research from 1980 to 2003 found that preferred sources varied by stage in the treatment process. Patients in the diagnosis and treatment phases tended to rely on print media and information from health professionals, whereas those in the post-treatment phase relied more on healthcare providers and interpersonal contacts for their information (Rutten et al., 2005).

Trust in health information gained from a variety of sources is also an essential driver of information-seeking. As with other aspects of the patient-provider relationship, trust in the provider affects the information exchange significantly. An environment allowing for participatory decision-making and information-sharing is often accompanied by, and may depend on, high levels of trust between the patient and provider (Kraetschmer et al., 2004). As mentioned earlier, patients often turn to individuals in their social networks for information, partly because of trust - this is an observation widely discussed in the literature surrounding social capital (Viswanath et al., 2006). We also know that cancer patients are interested in gaining information from the Internet, provided that the source is a reputable website (LaCoursiere et al., 2005). Other notable drivers of information-seeking regarding cancer are the beliefs the individual has about cancer diagnosis, treatment, and survivorship. For example, 
fatalism is often cited as a barrier to diagnosis and treatment among many individuals. (Reynolds, 2004; Wolff et al., 2003). For some patients, hope meant avid searching for information, especially about alternative treatments; but for others, it meant limited searching for or even avoidance of new information. Immediately after diagnosis, patients needed to be enabled to ask questions and seek information (Leydon et al., 2000). Besides, getting on with life and maintaining a positive outlook was perceived as the approach to managing the illness that was most respected by hospital staff, friends, and family. Even some found it easier to accept information obtained by friends and relatives because they had not personally used a scarce resource. Similarly, patients found information easier to accept when hospital staff verbally offered it rather than having to ask for additional information themselves (Leydon et al., 2000).

\section{METHODOLOGY}

\section{Research Context \& Sample}

We collected and analyzed area-specific female cancer patients in the Sidra Center, which is located in Badria Al-Ahmad hospital. This center serves patients at different stages of their fight against cancer, including: those who just discovered the disease and are not yet under treatment, patients that are taking treatment, and cancer survivors. The Sidra Center is considered as a health care provider that all cancer patients can continue to visit, especially to share their personal stories in the support group. The Sidra Center is considered as the main trusted and reliable information resource for most of its cancer patients. Our sample is focused on five female patients that have already finished their treatment and are thus called survivors. We are looking for their information-seeking behavior as survivors, exactly for the reasons that they avoid or lack the desire to seek or receive information regarding their disease. The reason behind choosing female survivors is that there are many studies which have confirmed that female patients are more likely to search for information related to their disease than males. Also, it is important to mention that the cancer survivors have specific characteristics for informationseeking, that they are younger and educated. Data was collected in two levels starting with four open-ended questions that will be filled out by us as researchers, because we found out that survivors are not interested in completing surveys other than the one in the Sidra Center. This approach offers a unique perspective on the perceptions of information-seeking patterns and behaviors for each patient. There is a difference between the information-seekers and nonseekers from different angles, including for example education, age, and information literacy.

\section{Research Instrument}

Interview is the method used in this research - as in much other healthcare research - to explore information-seeking behavior and different perspectives of cancer patients; this is because it provides in-depth analysis for researchers. The interview as a method gives a better way for interacting with the sample as they might enter new ideas, beliefs, issues, or concepts in the form of short sentences or symbols that they feel were important to the situation. The process starts by studying cancer survivors' information-seeking behaviors and looking for reason for not seeking information related to their disease. We attended their support groups and took notes on their interactions while chatting with others and with the psychologists. Then, the questions were answered by us while attending the support group for each of the five survivors. After that, each survivor was interviewed and their comments, answers and thoughts recorded for further 
analysis. This process of content analysis should help the emergence and tracking of new ideas, leading us to draw out the findings of this research. Analyzing the interviews in this way helped us to draw our findings in a way that will reach our research aims.

Different ideas were discovered after attending the first support group at the Sidra Center, such as that patients and survivors avoid health information because they have trust in their doctor's expertise. "Avoiding information is closely linked to feelings of anxiety and fear, as well as to other cognitive and emotional variables like perceptions of treatment efficacy, self-efficacy, and locus of control (the degree to which one's fate is governed externally versus controlled by one's self)" (Case, 2005). The psychologist at the Sidra Center was focused on a lot as the information source among cancer patients as they are one of the trusted resources, whereas friends and family members are not considered in the same way. In addition, the amount of information available on the Internet might mislead seekers and cause anxiety for non-seekers.

\section{FINDINGS \& DISCUSSION}

Cancer patients behave in two different ways for coping with their disease: either monitoring or blunting. As mentioned in the literature review above, blunting is our area of interest in this research, which means avoiding, ignoring, or lacking the desire for information-seeking. An interview carried out with five female cancer patients at the Sidra Center helped us to draw our findings. We have found that 3 out of 5 cancer survivors lacked the desire for receiving or seeking health information regarding their disease. This places these cancer patients under the health information non-seekers category. The interviews also assess and categorize patient's lack of desire into three main categories, as below.

\section{Online Information Resources (comparison between the use of online resources vs face-to- face)}

All interviewees stated that the Sidra Center for cancer information services is the best information resource they trust; they rely on it to take medical decisions and enhance their knowledge through. The second category in our findings indicates that information is available for cancer patients from healthcare providers, the Internet, libraries, the cancer information services (CIS), friends, and other sources (Zilinski, 2010). The Internet provides further health information for both seekers and non-seekers. It also provides health instructions, raises their information literacy and enhances their skills - this all can lead to immediate comfort and reduced anxiety. However, an issue emerged for us as researchers when interviewee five confirmed using an online search engine considered as a reliable information resource:

"Using Google is the best way for me to search for more in-depth details regarding my

disease, I like to be informed from a-z regarding my cancer, and get as much as needed of information from my doctor. I like when he answers all my questions and not hiding anything from me. I do not like to feel powerless and information give me power and make me relieved and I do feel no stress at all." 
We found that the reliability and quality of the information resource matters for cancer patients. "The knowledge gained from accessing online information and support and from participating in community and news venues of health websites can enhance one's self-efficacy and sense of empowerment" (LaCoursiere, 2005). Data from the Health Information National Trends Survey (HINTS) have also shown us that, while people say they would prefer to go to a healthcare provider for health information, most people actually use the Internet first.

Moreover, LaCoursiere's study demonstrated that cancer patients give attention towards other patients' experience. Interviewees four and five both verified that the effect of other patients' personal experience is great on self-efficacy and satisfaction towards the treatment:

"when other patients experience is being shared in the support group that is being held in

Sidra Center once a month, might enhance our social, emotional, and cognitive functioning, and resulted in greater satisfaction with treatment."

In addition, seeking online health information might lead to information overload and may increase stress and anxiety. Thus, the relationship between non-seeking cancer patients is very strong because of the trust given to the doctor as their main source of information. "Trust of online sources among cancer patients may be influenced by age, time since diagnosis, ability to cope with having cancer, and the perceived credibility of the source" (LaCoursiere, 2005). This confirms interviewee two and three's behavior - lacking the desire for information regarding their disease - as both of them rely on their doctor's advice and instructions while avoiding other sources of information:

“the best information resource for me is my doctor's instructions because I feel

comfortable and totally trust him. Also, the Sidra Center is another information resource that proven its role in the improvement of my health condition".

Therefore, it was found that although the use of online health care is available and increasing, obtaining health care information and support face-to-face remains the most frequent information preference for non-seeking cancer patients.

\section{The non-use of IS: Reasons}

The first category is related to reasons to behave as non-seekers, and to lack the desire for information; then we look at information resources and preferences for cancer patients; finally, we cover ways for motivating them to seek information and to change from non-seekers into information seekers. To emphasize, this research aims to understand the information non-seekers and develop a framework to help them in making health decisions. Ultimately, we aim to enhance the perfect strategy to reach their information preferences. Despite the studies that affirm that $52 \%$ of cancer patients had persistent information needs to look for health information and calm down after receiving it, we also found that $10 \%$ of cancer patients tend to 
avoid such information because it increased their anxiety. The perception of avoidance and lacking the desire for seeking information is rarely discussed in studies related to cancer information-seeking behavior. As Maslow once put it: "we can seek knowledge in order to reduce anxiety, and we can also avoid knowing in order to reduce anxiety".

\section{Fear appeals}

Starting with reasons behind lacking the desire for seeking information - or, in other words, blunting - chief among these is fear. "Research on 'fear appeals' considered another possibility: purposeful rejection of information" (Case et al., 2005). As the fourth interviewee confirmed, fear is a major reason for lacking the desire to seek or even receive information:

"At the beginning, I did not have the ability to hear or receive information regarding

my health condition, my cancer stage, and the treatment process because of fear".

After interviewing the five survivors in this study, it was found that cancer patients avoid threatening information or distract themselves from it for many reasons. These can include fear, attempts to control danger, becoming frustrated and depressed, anxiety, and trust in their doctor's health experience. This behavior was observed in each interviewee interaction especially after asking the third question, which is about the reasons for the lack of desire to look for information. The study of avoiding versus seeking by Case (2005) highlighted the importance of fear in relation to avoiding information: "High anxiety coupled with feelings of low self-efficacy are likely to invoke fear-control responses like denial, anger, guilt, or hopelessness."

\section{Trust}

Then, another reason for lacking the desire of receiving or seeking information could be believing and trusting that the doctor knows better. To corroborate this, we found that all five cancer survivors cite their physicians and doctors as the best source for information, as they would rather prefer receiving health information directly from them. The third interviewee clarified and insisted that it is better to allow the doctor to provide information about her disease and treatment and even make medical decisions for her. She said:

"I don't seek for information because my son is a doctor. I surely trust him and rely on health expertise and follow his instructions, he knows better".

Leydon (2000) indicates that the belief and trust cancer patients have in their doctor's experience can be represented as faith. "This was particularly the case among older patients, who, because they felt their knowledge and understanding of medicine was limited, believed that additional searching could be dangerous and exacerbate an already difficult situation." 


\section{Lack of internet access}

Also, cancer patients may lack the desire to seek information and avoid it simply because of the lack of access to the internet or bad network connection. Lack of access for some cancer survivors is the case of not having good and stable access; meanwhile, for others, it may be down to low information literacy and skills.

\section{Demographic influence}

Level of education and age are two crucial factors or reasons for lacking the desire to seek out health information. We found that demographic status has proven its role in influencing information-seeking; we concluded after the interviews that non-seekers are in this study between the ages of 45 and 64, and have not completed high school. Thus, higher education and higher income can be considered as factors leading someone to be in the information-seekers category, and vice versa for non-seekers. This perspective was clearly demonstrated when the second interviewee repeated the importance of awareness for educated women:

"Although we are educated and we got degrees, but we still not aware of our cancer causes, precautions, lifestyle, importance of periodic tests and examinations, and some general information. There is a need to spread awareness about cancer especially for the educated people, else what is the benefit of having a degree".

In addition, we found that language preference and beliefs regarding cancer can also be considered reasons for behaving as non-seekers. This is because it puts the patients under stress of understanding the difficult nature of information and avoiding it could be deemed the best for them. "If people do not believe that knowing more about a topic will allow them to effect a change, then they are not likely to seek information" (Case et al., 2005).

\section{Motivation: the real engine behind online searching}

We have found that if the interview with a cancer survivor begins with studies regarding cancer, they become more motivated to seek information. Also, they report more comfort and satisfaction at the mode of seeking information; they trust using other sources of health information. We have tended to start by leading with the main results from different studies, which indicate that: initially, the more you know the better medical decisions you will make. Then, the better decisions you make regarding your disease status, the better outcomes and immediate improvements would happen. Finally, knowing more and seeking information related to the process can reduce anxiety, lower depression, and raise self-efficacy. It was found that those three pieces of information were effective with cancer survivors and all five interviewees agreed. We start the final question in the interview with these three facts, to motivate cancer patients to change their lack of desire for information. As Zilinski (2010) demonstrated, "[p]atients who actively participate in decision-making and treatment options have been found to 
have less stress, stronger coping mechanisms, ask better questions of their physicians, seek out second opinions, and possibly participate in clinical trials and further research".

It was found that cancer patients are motivated by personal and social interactions, as interviewees four and five report. Moreover, a change in the lack of desire for seeking or receiving information could be influenced by several factors, including age, gender, ethnicity, and other demographic variables. Non-seekers are often found to be older - a factor which backs up Ramanadhan and Viswanath's report: "the choice of sources is mediated by gender, age, and education".

Also, interviewees one and five stated that the trust in their doctor's experience ranked top of their information resources, while online resources are also important. Interviewee one said:

"When there is not a way to go to the hospital because I do not have a care and it will cost me every time I come, searching online always represents a successful alternative

for me and satisfies my information needs".

Moreover, it was found that cancer survivors are motivated to seek information when they communicate with their support group team members for information; this resulted in helping them to better cope with their disease. As for interviewees 2, 3 and 4, refusals to ask for or receive information stemmed from an inside thought, whereby we find that it is difficult and overwhelming to place ourselves in a position to take decisions regarding their disease. Wilson's theory of "information behavior" was really helpful in understanding cancer patients while lacking the desire for seeking information. Wilson examined factors that evoked the need for information-seeking and reasons behind information resource preference.

Finally, to get cancer patients' attention and change this lack of desire, the issue of personal privacy or disclosure must be overcome. Similar findings by LaCorsiere (2005) and Rainie (2000) demonstrate that a lack of desire for seeking health information is highly connected to disclosure. So, if cancer patients feel comfortable with the information provider or resource, then better results will be displayed regarding the lack of desire to seek information in different resources. "Disclosure of personally identifiable health information is tightly regulated in regard to research situations and existing health care organizations" (LaCorsiere, 2005).

\section{CONCLUSION}

Despite the fact that cancer patients use a variety of information resources (such as the Internet, social media, and medical databases) to satisfy their needs, there are many non-seekers of information among cancer patients. Seeking behavior was analyzed in this research to determine cancer survivors' non-seeking of health knowledge. The purpose of this study was to investigate the information needs of cancer patients and to find out reasons for the lack of cancer stage knowledge and desire for information after diagnosis. The data were collected from patients at the Sidra Center, a facility that serves cancer patients at different stages of their disease, 
including: those who have just discovered the disease and are not yet under treatment, patients that are taking treatment, and cancer survivors.

Findings include feeling fear, trust in doctors, lack of internet access and demographic influence are the main reasons for information non-seeking among cancer patients. Our recommendations are summarized in motivating cancer patients to search online, starting with studies regarding cancer to utilize health information. Furthermore, we suggest applying some health orientation standards that would reduce anxiety, lower depression, and raise self-efficacy around information-seeking. Finally, cancer survivors become more motivated to seek information when they communicate with their support group's team members for information. Physicians and care providers strongly influence patients' decisions, and this can help them to better cope with their disease. We concluded that - despite the fact that the literature rarely discussed cancer patients lack of desire to seek information - there are some researchers interested in the non-seekers category. We also find that cancer patients' seeking behavior requires a particular attitude from physicians to study their coping strategy; more often than not, that's what defines their preferences for information. 


\section{REFERENCES}

Boudioni, M., McPherson, K., Mossman, J., Boulton, M., Jones, A. L., King, J., . . Slevin, M. L. (1998). An analysis of first-time enquirers to the Cancer BACUP information service: Variations with cancer site,demographic status and geographical location. British Journal of Cancer, 79, 138-145.

Brashers, E., Goldsmith, J., \& Hsieh, E. (2002). Information Seeking and Avoiding in Health Contexts. Human Communication Research, 28(2), 258-271.

Case, O., Andrews, E., Johnson, D., \& Allard, L. (2005). Avoiding Versus Seeking: the relationship of information seeking to avoidance, blunting, coping, dissonance, and related concepts. Journal of the Medical Library Association, 93(3), 353-362.

Carlsson, M. (2000). Cancer patients seeking information from sources out- side the health care system. Support Care Cancer, 8, 453-457.

Chou, Y., Prestin, A., Lyons, C., \& Wen, Y. (2013). Web 2.0 for health promotion: reviewing the current evidence. American Journal Public Health. 103(1), 9-18.

Costas-Muniz, R., Sen, R., Leng, J., Aragones, A., Ramirez, J., \& Gany, F. (2013). Cancer stage knowledge and desire for information: mismatch in Latino cancer patients. Journal Cancer Education, 28(3), 458-465.

Czaja, R., Manfredi, C., \& Price, J. (2003). The determinant and consequences of informationseeking among cancer patients. Journal of Health Communication, 8(6), 529-562.

Fox S. Health topics: $80 \%$ of Internet users look for health information online. Pew Internet $\&$

American Life Project Web site. http://www. pewinternet.org/reports/2011/healthtopics/part-

1/59-of-adults.aspx. Published February 1, 2011. Accessed November 25, 2012.

Hesse, W., Arora, K., Burke, E., \& Finney, J. (2008). Information Support for Cancer Survivors. American Cancer Society, 112(11), 2529-2540.

Kim, K., Lustria, M., Burke, D., Kwon, N. (2007). Predictors of cancer information overload: findings from a national survey. Information Research,12(4).1-29.

Koskan, A., Klasko, L., Davis, S. N., Gwede, C. K., Wells, K. J., Kumar, A., . . Meade, C. D. (2014). Use and Taxonomy of Social Media in Cancer-Related Research: A Systematic Review. American Journal of Public Health, 104(7), 20-37. 
Kraetschmer, N., Sharpe, N., Urowitz, S., \& Deber, R. B. (2004). How does trust affect patient preferences for participation in decision-making? Health Expectations, 7(4), 317-326.

Lacoursiere, S. P., Knobf, M. T., \& Mccorkle, R. (2005). Cancer Patients Self-Reported Attitudes About the Internet. Journal of Medical Internet Research, 7(3), 22.

Leydon, G. M. (2000). Cancer patients' information needs and information seeking behaviour: in depth interview study. Information in practice, 320(7239), 909-913.

Matthews, K., Sellergren, A., Manfredi, C., \& Williams, M. (2002). Factors influencing medical information seeking among African American cancer patients. Journal of Health

Communication, 7(3), 205-219.

Miller, M. (1987). Monitoring and blunting: Validation of a questionnaire to assess styles of information seeking under threat. Journal of Personality and Social Psychology, 52(2), 345-353.

National Cancer Institute (2007). Cancer Trends Progress Report-2007. Retrieved April 6, 2008, from https://hints.cancer.gov/docs/Briefs/HINTS_Brief_10_061708.pdf

Pinquart, M., \& Duberstein, P. R. (2004). Information needs and decision-making processes in older cancer patients. Critical Reviews in Oncology/Hematology, 5(1), 69-80.

Ramanadhan, S., Viswanath, K. (2006). Health and the Information Non-seeker: A profile. Health Communication, 20(2). 131-139.

Rees, C. E., \& Bath, P. A. (2000). The information needs and source preferences of women with breast cancer and their family members: A review of the literature published between 1988 and 1998. Journal of Advance Nursing, 31(4), 833-841.

Rees, E., \& Bath, A. (2001). Information-seeking behaviors of women with breast cancer. Oncology Nursing Forum, 28(3), 899-907.

Reynolds, D. (2004). Cervical cancer in Hispanic/Latino women. Clinical Journal of Oncology Nursing, 8(2), 146-150.

Rimer, K., Briss, A., Zeller, K., Chan, C., \& Woolf, H. (2004). Informed decision making: What is its role in cancer screening? Journal Cancer Education, 101(5), 1214-1228.

Rutten, J., Arora, K., Bakos, D., Aziz, N., \& Rowland, J. (2005). Information needs and sources of information among cancer patients: A systematic review of research (1980-2003). Patient Education and Counseling, 57(3), 250-261.

Serin, D. (2004). Parcours de Femme 2001: A French opinion survey on overall disease and everyday life management in 1870 women presenting with gynecological or breast cancer 
and their caregivers. Annals of Oncology, 15(7), 1056-1064.

Schou, I., Ekeberg, O., Sandvik, L., Hjermstad, M. J., \& Ruland, C. M. (2005). Multiple predictors of health-related quality of life in early stage breast cancer: Data from a year follow-up study compared with the general population. Qualitative Health Research, 14(8), $1813-1823$.

Viswanath, K., Breen, N., Meissner, H., Moser, B. P., Hesse, B., Steele, W. R., . . Rakowski, W. (2006). Cancer knowledge and disparities in the information age. Journal of Health Communication, 11(2), 1-17.

Viswanath, K., McCloud, R., Minsky, S., . . Emmons, K. M. (2013). Internet use, browsing, and the urban poor: implications for cancer control. Journal of the National Cancer Institute. Monographs. (47), 199-205.

Wolff, M., Bates, T., Beck, B., Young, S., Ahmed, S. M., \& Maurana, C. (2003). Cancer prevention in Underserved African American communities: Barriers and effective strategies-A review of the literature. Wisconsin Medical Journal, 102(5), 36-40.

Zilinski, L. (2010). Information Behaviors of Cancer Patients in the Information Age. Library Student Journal, 5. From http://www.librarystudentjournal.org/index.php/lsj/article/view/121/274 


\section{Appendix}

The goal of our interview is to reflect on your experience during the first stages of your diagnosis as a cancer patient:

1- When were you first diagnosed with cancer? What type of information did you look for? What type of resources did you use? Which resource did you prefer? (Probe on some stories of their search habits; let them talk about what they did, where they searched and how they search it. Stories and experiences.)

2- Focusing on each resource: (We want to do a comparison between each resource, and which one is most preferable and why.)

a. Search engines (Google): Why did you choose this resource? What kind of information were you looking for? What was the advantage and disadvantage of using this resource?

b. Social media: Why did you choose this resource? What kind of information were you looking for? What was the advantage and disadvantage of using this resource?

c. Specialized websites: (doctor's website, for example): Why did you choose this resource? What kind of information were you looking for? What was the advantage and disadvantage of using this resource?

d. Other online resources: Why did you choose this resource? What kind of information were you looking for? What was the advantage and disadvantage of using this resource?

e. Face-to-face (doctor, friends diagnosed with cancer, family member): Why did you choose this resource? What kind of information were you looking for? What was the advantage and disadvantage of using this resource?

We want to see if they have specific application, website, or software they use.

3- When did you STOP looking for information? At which stage? Why? What was the other alternative? Probe for STORIES

4- From your experience, what does a cancer patient need to better equip themselves with information to deal with health issues? How can we provide it to them? (Suggestions for improving their health information skills)

Before you leave the interview, make sure you got information related to:

1. Resources they use: Online VS offline

2. Their seeking behavior: how they search

3. The loss of desire to search: reasons behind their lost desire to know

4. How can we motivate them to search for information? What can we do to help? 